HLRZ preprint 46/93

Univ. Cologne Appl. Math. WP 93.133

\title{
Deterministic models for traffic jams
}

\author{
Kai Nagel ${ }^{+*}$, Hans J. Herrmann* \\ + ZPR and Math. Inst. der Universität, Weyertal 86-90, \\ 50923 Köln 41, Germany (permanent address) \\ kai@mi.uni-koeln.de \\ * HLRZ, c/o KFA, Pf. 1913, 52425 Jülich, Germany
}

May 24, 2022

\begin{abstract}
We study several deterministic one-dimensional traffic models. For integer positions and velocities we find the typical high and low density phases separated by a simple transition. If positions and velocities are continuous variables the model shows self-organized criticality driven by the slowest car.
\end{abstract}

\section{Introduction}

Everybody knows of the seemingly erratic motions of cars jammed on highways. One wonders whether they are due to a random behaviour of the individual drivers or if there is an intrinsic chaotic mechanism. In favour of the first hypothesis is the existence of regular kinematic waves in dissipative systems with excluded volume [1]. For this reason many traffic models include rather important statistical noise in time [2]. In favour of the second hypothesis are measurements performed on Japanese highways showing a $1 / f$ spectrum in the Fourier transformed density fluctuations [3] which might stem from some self-organized criticality [4]. It is therefore interesting to see if a traffic model without noise is able to give the observed erratic behaviour and its $1 / f$ spectrum.

In this paper, we systematically investigate deterministic variants of a simple one-dimensional model for traffic jams, which is, on the one hand, astonishingly realistic [2] and which can, on the other hand, be used for unprecedented high speed microscopic traffic simulations on parallel and/or vector computers [5]. Further analysis of the nondeterministic model may be found in [6, 7], and simplifications of this modeling technique have been applied to two-lane traffic [8] 
as well as to two-dimensional gridlock problems [9, 10, 11.

The deterministic model investigated in this paper shows, under certain circumstances, trivial critical behavior at a non-trivial critical density, and, providing enough input and output of mass at the boundaries, the open version of the model selects this density automatically. In addition, a straightforward extension of this model to floating point numbers leads to a deterministic system which exhibits non-trivial complex behavior even when starting from a totally ordered initial state.

\section{The prototype model}

The prototype of all models presented in this paper is a one-dimensional horizontal road with traffic flowing to the right of length $L$ (representing a road) with periodic boundary conditions. Each box may either be empty, or it may be occupied by a particle (vehicle) with an integer speed $0,1,2, \ldots$. A particle with velocity $v$ is either accelerated to $v \rightarrow v+1$ when there are at least $v+1$ empty boxes in front of the particle; or it is slowed down to $v \rightarrow k$ where $k$ is the number of empty boxes in front of the particle:

$$
\begin{array}{lll}
v \leq \Delta x-2 & \sim & v \rightarrow \Delta x-1 \\
v \geq \Delta x & \sim & v \rightarrow v+1,
\end{array}
$$

where $\Delta x=k+1$ is the gap between the particles plus the size of one particle. After the velocity update, the particle is advanced $v$ boxes to the right ("propagation step").

The original model as described in [2] goes beyond this model insofar as it introduces a maximum velocity (cf. further down in the present paper) and as it adds a high degree of noise to the dynamics taking into account the fluctuations of everyday driving behavior.

For the model presented here, three types of deterministic updates are possible: parallel, right circular, and left circular. Parallel means that all $N$ vehicles in the system are treated simultaneously. In this case, a vehicle cannot advance to more than one box behind the car in front has been the time step before. This update rule leads to gaps between particles proportional to their distance, which is realistic for traffic dynamics [12], and it therefore tends to disperse the particles (Fig. 1) as if they had a repulsive force of a range proportional to their speed.

"Right circular" update means that the update initially starts at some arbitrary particle (usually the leftmost one), adjusts its speed and performs its motion, and then proceeds with the next particle to the right and so on. When having periodic boundary conditions, this amounts to an "update demon" which circulates through the system. Nevertheless, for the "circular right" update it turns out that it is equivalent to the parallel update, with the following exception: At the position of the "update demon", it is possible to have two particles close together at high speed; in this case the left one just has been moved and the right one is the next particle to be moved. But this behavior does not change the mean properties for large system sizes. 
A "left circular" update, however, produces a markedly different dynamics. Here, a particle moves when the car in front already has moved away, which means that particles may move in clusters, each particle just catching up with the car in front during its update. In practice, any initial configuration leads after some time to only one cluster containing all vehicles, moving at speed $v_{\text {end }}=L-N$ (cf. Fig. 2). It is this update which we will denote as "circular" in the following.

\section{Transient times and damage times for the prototype model}

We measured each model's average "transient time" $\tau_{t}$, which is the time the system needs to settle down from a random initial condition to a regular steady state. Random initial condition here means that we placed the particles randomly and then drew integer velocities randomly between 0 and $L-1$ for each particle. ( $L-1$ is an upper bound for the velocity: Even in the case of only one particle in the system, when all update methods are equivalent, due to the periodic boundary conditions the particle "sees" itself at $\Delta x=L$ and can therefore not go faster than with $v=\Delta x-1=L-1$.)

With parallel update, the steady state is reached when the entire configuration of the system at time $t+1$ is a simple left shift of the configuration at time $t$ (as in the lower part of Fig. 1), which means that each particle assumes the velocity of the car in front. Nevertheless, already this simple model contains some of the features of a high traffic regime: If one follows the trajectory of one vehicle individually, regions of relatively free movement are alternating with regions of high density and slow speed. For the parallel update rule the average speed in the steady state equals the number of empty sites divided by the number of particles $\langle v\rangle=(L-N) / N$.

For the left circular update however, the steady state consists, as mentioned above, of only one cluster of particles which all move with the highest possible speed $L-N$.

In addition, we measured a "damage time" $\tau_{d}$ in order to estimate the duration of a disturbance 13. To do so, after reaching the steady state, we reduced the velocity of one randomly chosen particle by the smallest possible amount: $\tau_{d}$ is then the time the system needs to reach again a new steady state. Technically, for the parallel update, we reduced the velocity of the randomly chosen particle by one just before the propagation step, whereas we reduced it by two after the propagation step for the left circular update. In the latter case, a reduction by only one would have had no consequences at all.

Numerical results for $\tau_{t}$ as a function of the density $\rho=N / L$ are given in Fig. 3. We find for the parallel update and $L \rightarrow \infty$ the scaling $\tau_{t} \propto \rho^{-1}$ and $\tau_{d} \propto \rho^{-1}$, i.e. diverging times for the density going towards a "critical" density of zero.

The left circular update data (Fig. 4) shows no scaling behavior in $\rho$; and in fact there are simple explanations for the data: Since $\tau_{t}$ is simply the time the initially slowest particle needs 
to reach its final velocity, one has

$$
\tau_{t} \propto\left\langle v_{\max }\right\rangle-\left\langle\min \left(v_{\text {ini }}\right)\right\rangle=(L-N)-\frac{L}{(N+1)}=\frac{L-\rho L-1}{1+1 / \rho L} \sim L .
$$

The damage time $\tau_{d}$ is unity, when the leading particle is disturbed, and three otherwise. In average, this leads to $\tau_{d}=3-1 / N$.

\section{Introduction of a speed limit}

For real highway traffic as well as for dissipative systems under a constant force it is usually true that they have a limiting velocity even for the "free" particles which do not feel any influence of neighbors. Introducing a maximum velocity in our models simply means that a particle is only accelerated when it has not yet reached the maximum speed. We will use a maximum velocity of 5 throughout this article. It should be noted that only this introduction of the maximum velocity makes the particle interaction short-ranged.

When again starting from random initial conditions, this leads with parallel update for high densities to configurations similar to the case without velocity limit, but for low densities a new phase appears having clusters of vehicles moving with maximum velocity (Fig. 5). In other words, there are gaps between particles moving with maximum speed; a gap technically being defined as one or more sites not being crossed or touched by a particle during the propagation step. A left circular update, however, gives intermittent clusters of maximum velocity for all densities. As there is no velocity-dependent repulsive force between particles, many of the particles stick together.

The transient times for the parallel update case are plotted in Fig. 6. We find that at the non-trivial density $\rho_{c}=1 / 6$ the transient time $\tau_{t}$ shows a remarkable peak which grows with system size; finite size scaling analysis confirms that it diverges as

$$
\tau_{t}\left(\rho_{c}\right) \propto L
$$

The same is true for the damage time: $\tau_{d}\left(\rho_{c}\right) \propto L$. For the left circular update, the main change with respect to infinite velocity is that now even the transient time $\tau_{t}$ does no longer depend on $L$ for $L \rightarrow \infty$.

The phenomenological reason for the divergence at $\rho_{c}=1 / 6$ for the parallel update is as follows (explained for the damage time): At low densities, a disturbance only travels upstream to the next gap; the relevant length scale obviously depends on $\rho$ and not on $L$ (see Fig. 5). For high densities, however, (compare Fig. 1), the disturbance introduces a new wave front or reinforces an older one, and it leaves a gap traveling downstream because the disturbed particle cannot catch up quickly enough the car in front. This gap can only be filled in a region of particles which neither accelerate nor move at maximum speed, i.e., in a region with higher density. The length scale therefore depends on $\rho$. In between the high and the low density 
regimes, a transition takes place; and the critical point is a state where particles move with maximum velocity $v_{\max }=5$ and minimum distance $\Delta x_{\min }=v_{\max }+1=6$ (and therefore $\rho_{c}=1 / \Delta x_{\min }=1 / 6$ ). If one introduces a disturbance into this "critical" state, a fronts moves contrary to the flow direction until it meets downstream (periodic boundary conditions!) the gap caused by the disturbance before; hence the $L$-dependance of $\tau_{d}$ at $\rho_{c}$.

At the same time, $\rho_{c}$ is the density which corresponds to maximum particle throughput (current) $j=\rho v$. In fact, in the low density regime all particles have maximum speed $v_{\max }$ and therefore $j_{<}=v_{\max } \rho$, whereas in the high density regime the average speed is equal to the number of empty sites divided by the number of particles and therefore

$$
j_{>}=\frac{L-N}{N} \frac{N}{L}=1-\rho .
$$

These two straight lines intersect at $\rho_{c}=1 /\left(1+v_{\max }\right)$, which is therefore the density corresponding to maximum throughput.

For convenience, the different regimes are summarized in the following table:

$$
\text { parallel update } \quad \text { left circular update }
$$

\begin{tabular}{lll}
\hline$v_{\max }=L$ & $\begin{array}{l}\text { Tendency for equidistance, } \\
\text { waves. }(*)\end{array}$ & \\
$v_{\max }=5, \rho>\rho_{c}$ & $\begin{array}{l}\text { Tendency for equidistance; } \\
\text { waves with } v<v_{\max } . \text { Simi- }\end{array}$ & Tendency to cluster. \\
& lar to $(*)$. & $v_{\max } .(* *)$ \\
$v_{\max }=5, \rho<\rho_{c}$ & $\begin{array}{l}\text { Formation of gaps; no waves; } \\
\text { all cars } v_{\max } .\end{array}$ & Tendency to cluster, but not \\
& all cars; all cars $v_{\max }$. Simi- \\
& lar to $(* *)$.
\end{tabular}

\section{Self-organization of the critical state}

If one assumes a very dense jam as in Fig. 7, then the outflow from this jam assumes exactly the critical configuration of particles moving at maximum speed 5 and with a distance $\Delta x$ of 6 . This is no longer true for an open boundary (Fig. 8); but it can be restored, e.g. by forcing a higher acceleration for the first particle (Fig. 9). In this last case, a disturbance (as in Fig. 9) travels with constant speed to the left boundary, whereas the gaps travels with constant speed to the right boundary. Conceptually, this case of self-organized criticality therefore belongs to BTW's one-dimensional sandpile model $\llbracket$, with the difference that our model selects a state of non-trivial density.

With respect to reality, this means that the outflow of the traffic jam adjusts itself exactly at the critical density and therefore at maximum capacity, and due to the criticality, new disturbances have long-ranged effects through density waves traveling with constant speed. This may no longer be true for a bottleneck situation (e.g. a two-lane directional road merging into only one lane): The open boundary described in Fig. 8 leads to lower density and throughput, and the 
same is true for a more realistic model with strong noise (股, 8). Which of these findings exists in reality with its mixture of different vehicles and drivers is still an open question, although everyday experience tells us that traffic inside a bottleneck is indeed relatively stable and should therefore be below the critical density.

\section{A continuous version}

In order to investigate if our model shows more complex (and therefore more realistic) behavior when introducing a higher number of degrees of freedom, we considered a continuous version of our model. The one-dimensional system still has length $L$ with periodic boundary conditions; but velocity $v_{i}$ and position $x_{i}$ of a vehicle $i$ are now continuous variables. The update rule is as follows:

- If the velocity is high with respect to the gap, then the car slows down:

$$
v>\Delta x-\alpha \quad \leadsto \quad v \rightarrow \max (0, \Delta x-1) ;
$$

(the "max" is only necessary to prevent negative velocities);

- else if the velocity is low with respect to the gap and slower than five, then the car accelerates:

$$
v<\Delta x-\beta \quad \& \quad v<v_{\max } \quad \leadsto \quad v \rightarrow v+\min (1, \gamma * \Delta x) .
$$

Note that this rule allows maximum speeds up to nearly six.

- After the velocity has been updated for all vehicles according to the last two rules, we move all vehicles simultaneously according to their velocities.

In our simulation we used $\alpha=0.5, \beta=3.0$, and $\gamma=0.1$.

The only new feature of this model with respect to the integer version is that the acceleration is weaker when the distance is still small. A distinct feature of this model which it shares with the integer model is that there is a "dead zone" in the distance to the next car ahead where a driver neither accelerates nor slows down. This is in accordance with psychological investigations insofar as it stresses the importance of physiological thresholds in order to make human drivers react. Many car-following models use the velocity difference to the vehicle ahead instead of the gap as primary stimulus [12], and a practical implementation [14] uses thresholds which depend on the velocity difference and thresholds which account for the gap between two cars. And indeed, the latter are necessary in order to prevent grossly unrealistic behavior, allowing, e.g., very small gaps at very high velocities. However, suppression of the former, as we have done for our model, still leads to essentially realistic behavior.

With this model, we performed simulations of different setups. Whereas the normal closed systems showed a behavior similar to the integer model (i.e., settling down to an "imitate your 
leader"-state), already the introduction of one slightly slower vehicle leads to complex and unpredictable behavior. To be specific, the setup is as follows. In a system of length $L$ and with periodic boundary conditions, we initially placed $N=[\rho \cdot L]$ vehicles on sites 1 to $N$, all with velocity zero, where $\rho$ was chosen small enough to prevent any effect of the last car of the platoon on the first one through the periodic boundary conditions. (A platoon is an ensemble of vehicles travelling together.) Starting from this totally ordered initial state, the system was allowed to evolve according to the above rules, with the exception of the first vehicle, the speed of which was kept fixed at $v_{\text {lead }}=4.99999$ after its initial acceleration. This is a simplification of the well known situation where a number of fast vehicles has to follow a slower one which they cannot pass, and besides its obvious single-lane applications this situation also occurs on freeways when many passenger cars want to pass a group of trucks.

Fig. 10 shows a section of the evolution of the system. For a certain time, the density behind the first car gets larger because all vehicles close up. But at seemingly random instances, many of the followers have to slow down in one large collective event, which redistributes the vehicles with a lower density. As the first vehicle moves freely, the simulation represents, in spite of the periodic boundary conditions, the situation of a platoon moving infinitely in space.

In Fig.11 we see the time evolution of a system which had been transformed to the coordinates of the first vehicle, i.e. the positions of all cars are given relative to the first car. We see that equally spaced cars rapidly evolve into a fluctuating state (right hand side). In this new state density increases give rise to very short bursts (traffic jams) of very different sizes which redistribute the cars backwards such that in some cases they even start again in equally spaced patterns.

Although the model itself is totally deterministic, small perturbations may lead to totally different trajectories in phase space due to the chaotic dynamics. We have noticed this effect when simulating the same number of vehicles in systems of slightly different size: After a certain time, the development of the systems diverges, due to small differences in the transfer of cars through the periodic boundary. In order to clarify that this divergence is an intrinsic consequence of the dynamics and not just the enhancement of numerical round-off errors, we have compared single precision with double precision calculations. The overall result of these tests is that noise enters the system with the same "speed" for all cases, which is a strong indication that complex behavior originates from a chaotic dynamics and is not driven by the limited precision of the floating point numbers.

In addition, the principal behavior of the model (i.e. the formation of the collective shocks) is robust with respect to parameter changes. More precisely, we could not find a qualitatively different behavior for changes of the parameters $\alpha, \beta, \gamma$, and $v_{\text {lead }}$ within the following range $0.1 \leq \alpha \leq 0.6,2.0 \leq \beta \leq 5.0,0.08 \leq \gamma \leq 0.12,4.5 \leq v_{\text {lead }} \leq 4.99999$. 
In order to quantify these observations, we measured the distribution of times $\tau$ between consecutive "braking" events for the last vehicle ( $\tau$ is the time from the end of one braking to the beginning of the next). Braking is defined here as a slowing down according to the rules for the velocity update. We performed simulations on a Parsytec GCel-3, replicating a system with a fixed number of vehicles but different system sizes on up to 512 processors and averaging the results. For instance for $N=1900$ vehicles, we waited about $3 \cdot 10^{5}$ time steps to let the transients die out, and then measured the distribution of $\tau$ during about $1.1 \cdot 10^{6}$ further time steps. This specific simulation took about 33 hours on 256 processors.

According to Fig. 12, this distribution displays a remarkable straight line on a log-log-plot, fulfilling the power law

$$
n(\tau) \propto \tau^{-\alpha}
$$

with $\alpha=-2.2 \pm 0.1$. This non-trivial exponent is a strong indication for the existence of self-organized criticality 四 for this model.

Many aspects of this model are remiscent of the so-called train model for earthquake dynamics 15]. Instead of pulling at one end, the slower car may be seen as pushing against the other cars which want to move faster. This leads to a slowly increasing average density, and at some time this density locally exceeds a critical threshold. The reaction is a more or less drastic slowing down of the corresponding vehicle, which may or may not force the next vehicle to slow down as well. By this mechanism, avalanches of all sizes are generated, which may propagate through the entire platoon of vehicles.

\section{Conclusion}

We have studied several one-dimensional deterministic traffic models. As long as the velocities and positions are integers we find the well-known [2, 10], rather simple situation of a low density phase with particles having maximum velocity, a high density phase of low velocity waves and a simple transition between the two. When the velocity limit diverges the low density phase vanishes.

Much richer is a model in which continuous positions and velocities are allowed. If one car is a little slower we observe the spontaneous creation of erratic traffic jams of all sizes and a power law spectrum in the times between them. This self-organized behaviour is reminiscent of Burridge and Knopoff's original "train model" 15]. It would be interesting to analyze this model within the general theoretical considerations concerning self-organized criticality 16, 17].

\section{Acknowledgements}

We acknowledge computer time on the Parsytec GCel-3 of the ZPR (University of Cologne). Enlightening discussions with J. Kertész and M. Schreckenberg have helped us to beware of 
numerical round-off errors. KN thanks for discussions with and motivation by A. Bachem, J. Lee and M. Leibig.

\section{References}

[1] Leibig M, Pattern formation characteristics of interacting kinematic waves, preprint HLRZ$42 / 93$

[2] Nagel K, Schreckenberg M, A cellular automaton model for freeway traffic, J. Physique I 2 (1992) 2221.

[3] Musha $\mathrm{T}$ and Higuchi H, The $1 /$ f fluctuation of traffic current on an expressway, Jap. J. Appl. Phys. 15 (1976) 1271

[4] Bak P, Tang C, Wiesenfeld K, Self-organized criticality, Phys. Rev. A 38 (1988) 368.

[5] Nagel K, Schleicher A, Microscopic traffic modeling on parallel high performance computers, Appl. Math. and Comp. Sc. Work. Pap. 93.132 (1993), submitted to Parallel Comput..

[6] Schadschneider A, Schreckenberg M, Cellular automaton models and traffic flow, preprint 1993.

[7] Ito N, Nagel K, Schadschneider A, Schreckenberg M, in preparation.

[8] Nagatani T, Self-organization and phase transition in traffic-flow model of a two-lane roadway, 1993, submitted to J. Phys. A.

[9] Biham O, Middleton A, Levine D, Self-organization and a dynamical transition in trafficflow models, Phys. Rev. A 46 (1992) R6124.

[10] Cuesta J A, Martínez F C, Molera J M, Sánchez A, Phase transitions in two-dimensional traffic flow models, preprint 1993.

[11] Takayasu M and Takayasu H, 1/f noise in traffic, preprint 1993

[12] May A D, Traffic flow fundamentals, Prentice Hall, Englewood Cliffs, NJ, 1990.

[13] Kauffman S A, J. Theor. Biol. 22 (1969) 437.

[14] Wiedemann R, Simulations des Straßenverkehrsflusses, Schriftenreihe des Instituts für Verkehrswesen der Universität Karlsruhe, Heft 8, 1974.

[15] Sousa Vieira M de, Self-organized criticality in a deterministic mechanical model, Phys. Rew. A 46 (1992) 6288. 
[16] Krug J, Steady state selection in driven diffusive systems, in: Riste T, Sherrington D (eds.), Spontaneous formation of space-time structures and criticality, 37-40, Kluwer Academic Publishers, Netherlands, 1991.

[17] Carlson J M, Chayes J T, Grannan E R, Swindle G H, Self-organized criticality and singular diffusion, Phys. Rev. L 65 (1990) 2547. 


\section{Figure captions}

Fig. 1: Parallel update, $\rho=0.27, v_{\max }=L=70$. The entire highway is shown. Consecutive lines show configurations at consecutive time steps (i.e., time is evolving downwards), after the velocity update and before the propagation step. Empty spaces are marked by dots, particles by their integer velocities ("*” means that velocity is larger than 9). Particles move from left to right. The figure shows the evolution of the system from a random initial configuration to five steps after reaching the steady state and then the effect of a disturbance. Except for the first line, the same evolution could be possible for a system with $v_{\max }=5$.

Fig. 2: Left circular update, $\rho=0.3, v_{\max }=L=70$. The entire system is shown. The figure shows the evolution of the system starting from a random initial configuration.

Fig. 3: Transient times $\tau_{t}$ for $v_{\max }=\infty$ using parallel update. Systems of the same size are connected by lines.

Fig. 4: As Fig. 3, for left circular update.

Fig. 5: Parallel update, $v_{\max }=5, \rho=0.1$ (below $\rho_{c}$ ), $L=70$. This regime has no counterpart in the dynamics without speed limit.

Fig. 6: Transient times $\tau_{t}$ for $v_{\max }=5$ (parallel update). Systems of the same size are connected by lines.

Fig. 7: Deterministic outflow from a traffic jam.

Fig. 8: Acceleration from a dense situation (open system). Particles are inserted with maximum speed at the left boundary whenever there is space, but due to the other particles ahead they are immediately slowed down. In consequence, the system is not able to reach its maximum throughput.

Fig. 9: Acceleration from a dense situation (open system) with a forced acceleration of the leftmost particle. This means that at this position, a particle instantly can accelerate from zero to, e.g., the maximum speed five (see, e.g., from the first to the second line).

Fig. 10: Evolution of the continuous model (5600 time steps), $N=61, L=1024$. As in the pictures for the deterministic system, consecutive lines represent configurations at consecutive time steps; vehicles are denoted by black squares, empty spaces are left blank.

Fig. 11: First 4200 time-steps of the continuous model, transformed to the coordinates of the leading (rightmost) vehicle. Only every third time-step is plotted, everything else is as in Fig. 10. The jam-waves are now clearly visible as nearly horizontal lines. The flow is from left to right.

Fig. 12: Distribution $n(\tau)$ of times $\tau$ between consecutive braking events for $N=190$ and $N=1900$ cars. (The times are collected in "logarithmic bins", so that the $y$-axis is proportional to $\tau n(\tau)$.) 